\title{
Functional approach to the electromagnetic response function: the Longitudinal Channel
}

\author{
R.Cenni and P.Saracco \\ Dipartimento di Fisica dell'Università di Genova \\ and I.N.F.N. - Sez. Genova, Via Dodecaneso,33 - 16146 Genova (Italy)
}

(April 9, 2018)

In this paper we address the (charge) longitudinal electromagnetic response for a homogeneous system of nucleons interacting via meson exchanges in the functional framework. This approach warrants consistency if the calculation is carried on order-by-order in the mesonic loop expansion with RPA-dressed mesonic propagators. At the 1-loop order and considering $\pi, \rho$ and $\omega$ exchanges we obtain a quenching of the response, in line with the experimental results.

21.65

\section{INTRODUCTION}

The inclusive cross section for electron scattering off a nuclear target reads

$$
\begin{aligned}
\frac{d \sigma}{d E^{\prime} d \Omega}=\sigma_{M} & \left\{\frac{Q^{4}}{|\mathbf{q}|^{4}} R_{L}\left(\omega, Q^{2}\right)\right. \\
+ & {\left.\left[\frac{Q^{2}}{2|\mathbf{q}|^{2}}+\tan ^{2} \frac{\theta}{2}\right] R_{T}\left(\omega, Q^{2}\right)\right\} }
\end{aligned}
$$

and allows the experimental separation of the longitudinal and transverse electromagnetic response functions $R_{L / T}$ by means of a Rosenbluth plot of data taken at the same $Q^{2}$ and different angles.

Such separation is grounded on two approximations, namely the one-photon-exchange and the negligibility of Coulomb distortion of the electrons. In the inclusive cross section the two photons exchange corrections are of order of $1 \%$ [ [ ] and may be safely neglected. Instead, the use of different kinematics, differently affected by the Coulomb distortion, could make the separation somehow questionable 2 月. 4 .

Even within these limits, however, the Rosenbluth separation of $R_{L / T}$ has recently become available for a wide variety of nuclei, in different energy and momentum regions [5 13] and has provided new, unexpected outcomes: the central problem seems to be that a Free Fermi Gas (FFG) model is roughly able to reproduce the transverse response $-2 \mathrm{p}-2 \mathrm{~h}$ corrections improve the agreement with the experimental data [14] within a mesonic theory; on the contrary, the FFG model prevision in the longitudinal channel is almost twice as large with respect to the experimental results. The most recent data on ${ }^{40} \mathrm{Ca}$ 13] seem to weaken this conclusion: we will come back to this point later on.
Many attempts to simultaneously explain both the responses have been carried out - we restrict our considerations to heavy and medium nuclei - but the results of these theoretical efforts are still largely unsatisfactory. Both details and a critical discussion about them can be found, for instance, in Ref. [15].

At lower $q$ calculations performed along the RPA or Tamm-Dancoff approximation schemes improve the agreement with the data 1620 as far as collectivity can strongly influence the response. However, at momenta of the order of 300 or $400 \mathrm{MeV} / \mathrm{c}$ this mechanism cannot be effective anymore, but still the discrepancy between the experimental data and the theory survives.

An interesting suggestion comes from the bag model: a partial deconfinement mechanism could be effective inside nuclear matter, leading to the so-called "swollen nucleon hypothesis": the corresponding change in the e.m. form factor could well account for the quenching of the quasi elastic peak (QEP) [21 23]; the experimental outcomes in the transverse channel remain, however, unexplained: if the nucleon is swollen, $R_{T}$ should be quenched too. A possible solution was suggested by M. Ericson and Rosa-Clot [24]: in a mesonic model the swollen nucleon can be seen as a bare nucleon surrounded by its mesonic cloud, now feeling the presence of the other nucleons of the medium so affecting in some unspecified way the photo-absorption. It is easy to see that such a mechanism is channel dependent, giving us a chance of explaining both the longitudinal and transverse response functions in the frame of a mesonic theory.

We shall investigate this last topic in detail, examining in the present paper the longitudinal response function; we plan, in two other following papers, to study the transverse response and the nuclear sum rules as well.

Two crucial points need to be clarified just from the beginning, in order to give a defined content to the words "mesonic theory": by one side a complete specification of the dynamical model is needed - this means to decide which kinds of mesons and baryons are considered as true degrees of freedom, to establish a lagrangian governing their dynamics and, furthermore, to discuss which other quantities need to be parametrized to obtain a realistic description of the system; on the other side, a well defined theoretical scheme is asked for, able to select which approximations can be safely carried out to obtain a practically manageable and well behaved expansion without violating general theorems.

Few years ago we proposed a scheme based on the Sad- 
dle Point Approximation (SPA) applied to the generating functional for a system of nucleons and pions [25]: this step can be performed after the explicit integration of the fermionic degrees of freedom; in the resulting expansion the ring-dressed meson propagators are the mean field level of the theory, the higher order corrections being expressed in terms of a loop expansion with respect to these ring-dressed boson propagators.

In 25] we concentrated on a relativistically covariant $\pi-N$ system, but the expansion there derived was characterized by the topology of the diagrams: thus we are free - as we shall prove in the following - to apply the same scheme to the non relativistic restriction of the model and to mesonic fields other than the pionic one.

In the present paper we shall include into the model both the $\rho$-meson, displaying a remarkably different behaviour in the spin-transverse channel, and the $\Delta_{33}$ resonance, whose contribution is relevant to a realistic description of the nuclear dynamics. This will provide both the theoretical framework for a forthcoming paper and a self-contained explanation of the approach.

The results of a preliminary calculation were presented in [26] in a purely pionic framework. The theoretical expectation of strong cancellations in the selected class of diagrams was supported by the almost complete suppression of the one-loop corrections in the isovector channel; however the longitudinal response was still overestimated.

In the present paper we extend the previous calculations to a richer dynamical model by allowing the exchange of $\rho$ and $\omega$ mesons and the excitation of $\Delta$ resonances in the intermediate states. These contributions significantly improve the agreement with the experimental data.

The paper is organized as follows: in sect. II the bosonic loop expansion is presented and the one-loop corrections are derived. The formalism is sufficiently general to apply to the transverse channel as well, but the Meson Exchange Currents (MEC) are not considered in the present paper: they will be discussed when investigating the transverse (magnetic) response. In sect. III the dynamical model will be presented and discussed. In sect. IV we shall illustrate the details of the calculation and in sect. $\mathrm{V}$ the results will be presented. Finally in sect. VI we shall comment and discuss our outcomes in connection with the experimental data. Some outlooks on the perspectives of the present work are also presented.

\section{THE BOSONIC LOOP EXPANSION}

The functional approach to the nuclear Many-Body problem was repeatedly described in previous works 25,27 29, the central idea being the projection of the action on a bosonic Hilbert space via a functional integration of the fermionic degrees of freedom. We studied in details in 25] the e.m. response function within the functional approach for a system with nucleons and pions only. Here we introduce two main modifications, namely the non relativistic approximation and the inclusion of those short range correlations (SRC) not coming from the exchange of the mesons actually present in the model, to allow the use of the presently available phenomenological models of low momentum strong interactions, like, e.g., the Bonn potential or whatever else mesonic theory.

To preserve coherence as far as possible, but still remaining sufficiently general, we confine ourselves to a potential theory with local interaction in the various particle-hole channels with assigned spin, isospin and helicity.

The classical action (in terms of Grassmann variables) takes the form

$$
\begin{aligned}
A= & \int d x d y \psi^{\dagger}(x) G_{0}^{-1}(x-y) \psi(y) \\
& -\frac{1}{2} \int d x d y \sum_{i} \psi^{\dagger}(x) \Gamma_{i} \psi(x) V_{i}(x-y) \psi^{\dagger}(y) \Gamma_{i} \psi(y)
\end{aligned}
$$

where the $\Gamma_{i}$ denote the relevant spin-isospin matrices (to exemplify, in the spin-longitudinal isovector channel $\Gamma_{i}=$ $\left.-i \boldsymbol{\sigma} \cdot \boldsymbol{\nabla} \tau_{i}\right)$ for which we require a sort of orthogonality, namely

$$
\operatorname{Tr} \Gamma_{i} \Gamma_{j}=n_{i} \delta_{i j} .
$$

We next couple the system to some external classical fields $J_{i}$ with the same quantum numbers of the corresponding $\mathrm{p}-\mathrm{h}$ channels and write the generating functional as

$$
Z\left[J_{i}\right]=\int \mathcal{D}\left[\psi^{\dagger}, \psi\right] e^{i A+i \sum_{i} \psi^{\dagger} \Gamma_{i} \psi J_{i}}
$$

the response function in a given channel follows from

$$
\Pi^{i}(x, y)=-\left.\frac{\delta^{2} \log Z[J]}{\delta J_{i}(x) \delta J_{i}(y)}\right|_{J_{i}=0}
$$

and

$$
R_{i}(q)=-\frac{1}{\pi} \Im \int d^{4}(x-y) e^{i q \cdot(x-y)} \Pi^{i}(x-y) .
$$

The thermodynamic limit is here understood, but it is not in principle required: the present scheme, with straightforward modifications, could apply to a finite system as well; however the required computational effort makes an actual calculation realistically not feasible.

The projection on a bosonic Hilbert space is carried out by means of a Hubbard-Stratonovitch transformation 30 32, i.e., by exploiting the identity

$$
\begin{aligned}
& e^{-\frac{i}{2} \int d x d y \psi^{\dagger}(x) \Gamma_{i} \psi(x) V_{i}(x-y) \psi^{\dagger}(y) \Gamma_{i} \psi(y)}= \\
& \sqrt{\operatorname{det} V_{i}} \int \mathcal{D}\left[\sigma_{i}\right] e^{\frac{i}{2} \int d x d y \sigma_{i}(x) V_{i}^{-1}(x-y) \sigma_{i}(y)} \\
& \times e^{i \int d x \sigma_{i}(x) \psi^{\dagger}(x) \Gamma_{i} \psi(x)},
\end{aligned}
$$


then substituting (7) into (估) and carrying out the remaining gaussian integration over the fermionic fields. We end up (after a shift in the integration variable $\sigma_{i}$ ) with

$$
Z\left[J_{i}\right]=\exp \left\{\frac{i}{2} \sum_{i} J_{i} V_{i}^{-1} J_{i}\right\} \int \mathcal{D}[\sigma] \exp \left\{i A_{\text {eff }}^{B}\right\}
$$

where

$$
\begin{gathered}
A_{\mathrm{eff}}^{B}=\frac{1}{2} \sum_{i} \sigma_{i} V_{i}^{-1} \sigma_{i}-i \operatorname{Tr} \sum_{n=1}^{\infty} \frac{1}{n}\left[\sum_{i} \sigma_{i} \Gamma_{i} G_{0}\right]^{n} \\
-\sum_{i} \sigma_{i} V_{i}^{-1} J_{i} .
\end{gathered}
$$

This effective action has been derived in the frame of a potential theory. However, a mesonic model leads to the same form of the generating functional with the potentials $V_{i}$ replaced by meson propagators; the fields $\sigma_{i}$ can then be interpreted as the true mesonic fields. Thus, thanks to this unifying aspect of the formalism, we can proceed without distinguishing between mesonic and potential theories and attribute to the $V_{i}$ 's the appropriate meaning, potential or meson propagator, directly when needed. In eqs. (8) and (9) the quantity $V_{i}^{-1}$ appears, so raising the delicate question of its existence: this problem can be formally solved by means of the change of variable $\sigma_{i} \rightarrow V_{i} \sigma_{i}$ in the functional integrals, but we prefer to keep the present form to preserve to $\left\langle\sigma_{i}\right\rangle$ the meaning of expectation value of the underlying mesonic fields.

The next step is the evaluation of the last path integral in eq. (8). We adopt the semiclassical expansion, i.e., the path integral is evaluated within the Stationary Phase Approximation - or Saddle Point Approximation in the Euclidean word, in both cases SPA. This amounts to impose the stationarity of the effective action $A_{\text {eff }}^{B}$ with respect to arbitrary variations of the fields $\sigma_{i}$ :

$$
\begin{gathered}
J_{j}(x)=\sigma_{j}(x) \\
-\operatorname{Tr} \int d y V_{j}(x-y) \sum_{n=1}^{\infty} \int d y_{1} \ldots d y_{n} G_{0}\left(y-y_{1}\right) \\
\cdot \sum_{i} \Gamma_{i} \sigma_{i}\left(y_{1}\right) G_{0}\left(y_{1}-y_{2}\right) \ldots \sum_{k} \Gamma_{k} \sigma_{k}\left(y_{n}\right) \\
\times G_{0}\left(y_{n}-y\right) \Gamma_{j} .
\end{gathered}
$$

The solutions of eq. (10) are clearly functionals of the external sources $J_{j}$; they will be denoted from now on by $\sigma_{j}^{\mathrm{RPA}}$ because of the link, that will become clear in the following, with the Random Phase Approximation or more precisely, with the ring approximation, as it is sometime referred to.

A peculiar role is played by the scalar-isoscalar field (if any), because it brings into the theory the only possible tadpole; if so, a spontaneous symmetry breaking occurs and a shift in the corresponding integration variable is needed to force back the field expectation value to zero; this procedure entails a finite renormalization of the non interacting nucleon Green's function $G_{0}$ with the Hartree potential generated by the tadpole. In the present work we do not introduce the $\sigma$-meson: then this procedure is not needed.

We can solve the SPA equation in the form of Volterra expansions for $\sigma_{j}^{\mathrm{RPA}}$ as functional of $J_{j}$. Having ruled out the term with $n=1$ in eq. (10), the $0^{\text {th }}$-order term of the expansion vanishes and we can write 7 :

$$
\begin{gathered}
\sigma_{i}^{\mathrm{RPA}}(x)=\sum_{k} \int d y A_{k}^{i}(x \mid y) J_{k}(y)+ \\
+\frac{1}{2} \sum_{k m} \int d y d z B_{k m}^{i}(x \mid y, z) J_{k}(y) J_{m}(z)+\mathcal{O}\left(J^{3}\right) \\
\equiv A_{k}^{i}(x \mid y) J_{k}(y)+\frac{1}{2} B_{k m}^{i}(x \mid y, z) J_{k}(y) J_{m}(z) \\
+\mathcal{O}\left(J^{3}\right)
\end{gathered}
$$

Substituting the form (11) into (10) and collecting the terms linear in $J_{i}$ one gets for $A_{k}^{i}$ the equation

$$
\begin{aligned}
A_{k}^{i}(x \mid y)=n_{i} \int & d z d t V_{i}(y-z) \Pi^{(2)}(z-t) A_{k}^{i}(t \mid y) \\
+ & \delta_{i k} \delta(x-y)
\end{aligned}
$$

$\Pi^{(2)}$ denoting the Lindhard function. The reasons of this unusual notation will become clear later, see eq (21). Then, since the RPA-dressed potential (or meson propagator) reads

$$
\Delta_{\mathrm{RPA}}^{i}=\frac{1}{V_{i}^{-1}-n_{i} \Pi^{(2)}}
$$

the solution for $A_{k}^{i}$ takes the form

$$
A_{k}^{i}(x \mid y)=\Delta_{\mathrm{RPA}}^{i}(x-z) V_{i}^{-1}(z-y) \delta_{i k} .
$$

It is remarkable that formally

$$
A=\frac{1}{1-n V \Pi^{(2)}}
$$

so that if no interaction in the $j^{\text {th }}$ channel is allowed $\left(V_{j}=0\right)$, then

$$
A_{k}^{j}(x \mid y)=\delta_{j k} \delta(x-y) .
$$

The equation for $B$ reads

$$
\begin{gathered}
B_{j k}^{i}(x \mid y, z)=n_{i} V_{i}(x-u) \Pi^{(2)}(u-v) B_{j k}^{i}(v \mid y, z)+ \\
2 \operatorname{Tr} \Gamma_{i} \Gamma_{j} \Gamma_{k} V_{i}(x-u) G_{0}(u-v)\left(\Delta_{\mathrm{RPA}}^{j} V_{j}^{-1}\right)(v-y) \\
\cdot G_{0}(v-t)\left(\Delta_{\mathrm{RPA}}^{k} V_{k}^{-1}\right)(t-z) G_{0}(t-u)
\end{gathered}
$$

with the immediate solution

\footnotetext{
${ }^{1}$ We use, when no confusion can arise, the convention that a sum is understood when some spin-isospin index is repeated two and only two times. In the same way a repeated spacetime variable is meant to be integrated.
} 


$$
\begin{array}{r}
B_{j k}^{i}(x \mid y, z)=2 \operatorname{Tr} \Gamma_{i} \Gamma_{j} \Gamma_{k} \Delta_{\mathrm{RPA}}^{i}(x-u) G_{0}(u-v) \\
\cdot\left(\Delta_{\mathrm{RPA}}^{j} V_{j}^{-1}\right)(v-y) G_{0}(v-t) \\
\cdot\left(\Delta_{\mathrm{RPA}}^{k} V_{k}^{-1}\right)(t-z) G_{0}(t-u) .
\end{array}
$$

Having solved the SPA equations, the generating functional reads

$$
\begin{aligned}
Z\left[J_{j}\right]=e^{\frac{i}{2} \sum_{i} J_{i} V_{i}^{-1} J_{i}} e^{i A_{\mathrm{eff}}^{B}\left[\sigma_{j}^{\mathrm{RPA}}\right]} & \times \operatorname{det}\left\{\left.\frac{\delta^{2} A_{\mathrm{eff}}^{B}[\sigma]}{\delta \sigma_{i}(x) \delta \sigma_{j}(y)}\right|_{\sigma_{k}=\sigma_{k}^{\mathrm{RPA}}}\right\}^{-\frac{1}{2}}
\end{aligned}
$$

where the exponent describes the mean field propagation while the determinant is responsible for the one-loop (quadratic) corrections [both discrete and continuous indices must be considered in the evaluation of the symbolic determinant in Eq. (19)].

Let us consider first of all the mean field response functions: to obtain the linear response it suffices to replace into $A_{\text {eff }}^{B}$ the SPA solutions for $\sigma_{i}$ up to the first order in $J_{i}$; only the term with $n=2$ of the sum in (9) comes into play and then

$$
-\left.\frac{\delta^{2} \log Z}{\delta J_{i} \delta J_{i}}\right|_{J_{i}=0}=\frac{\Pi^{(2)}}{1-n_{i} V_{i} \Pi^{(2)}}
$$

i.e., the response function is given by its RPA approximation.

When the potential is vanishing the mean field response reduces to the FFG one. To exemplify, in the case we are interested in - the response to a scalar-isoscalar plus scalar-isovector probe, a charge longitudinal $\gamma-$ and if the effective interaction carries only the $\pi, \rho$ or $\omega$ quantum numbers both the mean fields turn out to coincide with the FFG ones. Instead, in the spin transverse channel (the magnetic one) the $\rho$-meson propagation is able to dress at the mean field level the FFG response.

Let us now come to the one-loop corrections: the fermionic loops up to $n=4$ are relevant. We define, following the notations of [33,34], the functions

$$
\begin{aligned}
\Pi^{(2)}(x, y) & =G_{0}(x-y) G_{0}(y-x) \\
\Pi^{(3)}(x, y, z) & =G_{0}(x-y) G_{0}(y-z) G_{0}(z-x) \\
\Pi^{(4)}(x, y, z, t)= & G_{0}(x-y) G_{0}(y-z) G_{0}(z-t) \\
& \times G_{0}(t-x),
\end{aligned}
$$

which are symmetric for cyclic permutations of the arguments: the second derivatives of $A_{\text {eff }}^{B}$ result:

$$
\begin{gathered}
\frac{\delta^{2} A_{\text {eff }}^{B}}{\delta \sigma_{i}(x) \delta \sigma_{j}(y)}=V_{i}^{-1}(x-y) \delta_{i j}- \\
\sigma_{k}(z)\left\{\Gamma_{i} \Gamma_{j} \Gamma_{k} \Pi^{(3)}(x, y, z)+\Gamma_{j} \Gamma_{i} \Gamma_{k} \Pi^{(3)}(y, x, z)\right\} \\
-\sigma_{k}(z) \sigma_{m}(t)\left\{\Gamma_{i} \Gamma_{j} \Gamma_{k} \Gamma_{m} \Pi^{(4)}(x, y, z, t)+\right. \\
\left.\Gamma_{j} \Gamma_{i} \Gamma_{k} \Gamma_{m} \Pi^{(4)}(y, x, z, t)+\Gamma_{i} \Gamma_{k} \Gamma_{j} \Gamma_{m} \Pi^{(4)}(x, z, y, t)\right\} .
\end{gathered}
$$

Next using the well known property

$$
\operatorname{det} X=e^{\operatorname{Tr} \log X}
$$

the one loop contribution to the polarization propagator becomes

$$
\begin{aligned}
\Pi_{i}^{1-\text { loop }}(x, y)=- & \frac{i}{2} \frac{\delta^{2}}{\delta J_{i}(x) \delta J_{i}(y)} \\
& \cdot\left[\left.\operatorname{Tr} \log \frac{\delta^{2} A_{\mathrm{eff}}^{B}}{\delta \sigma_{i}(x) \delta \sigma_{j}(y)}\right|_{\sigma_{i}=\sigma_{i}^{\mathrm{RPA}}}\right]_{J_{k}=0} .
\end{aligned}
$$

The logarithm is required up to the order $J^{2}$ : thus we multiply in the exponent by $V_{i}-$ a quantity not affecting the response because independent from $J$ - and we expand the logarithm up to the second order before deriving it with respect to the external sources. A tedious but straightforward calculation provides

$$
\begin{gathered}
\Pi_{i}^{1-\operatorname{loop}}(x, y)=i\left(\Delta_{\mathrm{RPA}}^{i} V_{i}^{-1}\right)(x-z) \operatorname{Tr} \Gamma_{i} \Gamma_{k} \Gamma_{i} \Gamma_{k} \\
\Pi^{(4)}(z, u, t, v) V_{k}(u-v)\left(\Delta_{\mathrm{RPA}}^{i} V_{i}^{-1}\right)(t-y) \\
+i\left(\Delta_{\mathrm{RPA}}^{i} V_{i}^{-1}\right)(x-z) V_{k}(u-v) \operatorname{Tr} \Gamma_{i} \Gamma_{k} \Gamma_{k} \Gamma_{i} \\
\left\{\Pi^{(4)}(z, u, v, t)+\Pi^{(4)}(t, u, v, z)\right\}\left(\Delta_{\mathrm{RPA}}^{i} V_{i}^{-1}\right)(t-y) \\
+i\left(\Delta_{\mathrm{RPA}}^{i} V_{i}^{-1}\right)(x-z) \operatorname{Tr} \Gamma_{i} \Gamma_{j} \Gamma_{k} \Pi^{(3)}(z, t, u) V_{j}(t-v) \\
V_{k}(u-w)\left\{\operatorname{Tr} \Gamma_{i} \Gamma_{k} \Gamma_{j} \Gamma_{j} \Pi^{(3)}(s, w, v)\right. \\
\left.+\operatorname{Tr}_{i} \Gamma_{j} \Gamma_{k} \Pi^{(3)}(s, v, w)\right\}\left(\Delta_{\mathrm{RPA}}^{i} V_{i}^{-1}\right)(s-y) .
\end{gathered}
$$

The corresponding Feynman graphs are plotted in fig. 1.

The theory, in the form here presented, has been developed for a system of nucleons interacting either through a potential or through a meson exchange, without explicitly including the possible excitation of baryonic resonances. This drawback is overcame by replacing the fermionic fields with fermionic multiplets, whose components are the true field operators for the various kinds of baryons. The diagrams so generated are topologically identical to those of fig. 11. but each fermionic line stands now either for a nucleon or for a $\Delta$, or for whatever else resonance we choose to include into the dynamical model.

\section{THE DYNAMICAL MODEL}

The approximations here described are sufficiently general to apply to various dynamical schemes. We now describe the one we are going to follow.

The complete determination of the model requires the specification of two ingredients - the form of the current and the potential we choose.

Concerning the first point, we recall that the response to an e.m. probe in the longitudinal channel is usually described, in a non relativistic context, by its nucleonic part, neglecting any contribution coming from the MEC [contact term, pion-in-flight, direct $\Delta$-excitation]. This 
assumption seems quite acceptable, and we shall follow it in this paper: in principle, however, we cannot exclude a significant contribution coming from the MEC, even in the longitudinal channel, when relativity is correctly accounted for. In the one-loop approximation, in fact, we expect large cancellations between the various contributions - and they were found indeed in the isovector channel in a preliminary calculation [26]: in such a case even small contributions could become relevant. Thus the corrections coming from the MEC in the longitudinal channel are for the moment neglected, but they will be object of a future investigation.

To study the longitudinal response we only need two external sources, namely $\Gamma_{T=0}=I$ and $\Gamma_{T=1}=\tau_{3}$, the full response being at the FFG level, with obvious notations,

$$
R_{L}=\frac{1}{4} R_{T=0}+\frac{1}{4} R_{T=1}
$$

Both the response functions in (24) contain, of course, the usual Sachs form factor $G_{E p}$.

Next we come to the second, and more delicate, problem, namely the choice of the $V_{i}$. Three mesonic fields are known to dominate the nuclear dynamics - as it can be argued for instance from the $N-N$ phase shifts analysis leading to the Bonn potential [35, 36] - namely those of $\pi, \rho$ and $\omega$ mesons. To follow this scheme we shall only consider those $V_{i}$ pertaining to the isovector spin-longitudinal and spin-transverse channels and to the isoscalar spin-transverse one. This does not mean that we completely neglect the effective interaction in other channels, which should be thought as arising, largely, from the two mesons exchange, a process accounted for in our scheme by the diagrams d) and e) of fig. 1. It is important to remember, for instance, that the $\sigma$ meson - carrying the scalar-isoscalar part of the interaction - was introduced in the past to simulate the exchange of two pions with the simultaneous excitation of one (or two) of the intermediate nucleons to a $\Delta$. In the present scheme these contributions - often referred to as the "box diagrams" in the language of the Bonn potential - are explicitly accounted for.

We now examine the model interaction in the various channels, starting from the isovector spin-longitudinal one. There, since the pioneering works of Migdal on pion condensation [37,38], the effective interaction is described by the one pion exchange plus a short range contribution schematized by the Landau parameter $g^{\prime}$ which simulates "whatever else can happen" in that channel [39 41]. In the present scheme this assumption is too poor for at least two reasons: the first is that in an one loop calculation a constant potential leads to (obviously spurious) divergences, and the same happens for a $\mathrm{p}$-wave static pion exchange potential (see Ref. [42] for more details); the usual $\pi N N$ vertex form factor obviously removes the divergence, but it leaves unaltered the unphysical high momentum behaviour of the interaction, which in this way is simply hidden. The second reason is that, again at the one loop level, some diagrams can occur with two consecutive Landau effective interactions attached to the same fermionic line [this is immediately seen to happen, again, for diagrams d) and e) of fig. 1, but the same is also true for diagrams b) and c)]: this means that some double counting can, in principle, be present in the calculation; thus we must spend some more words to better specify the physical meaning we attribute to $g^{\prime}$ at the 1-loop level.

When the momentum carried by one meson [or potential] line is not limited by the kinematics, as instead it happens at the mean field [0-loop] level, it is necessary to account for a major effect, usually referred to as "short range correlations" [SRC] [43]: they prevent, for instance, the pion to be exchanged between nucleons too near the one to the other. This effect can be ascribed, within a mesonic theory, to a short range repulsive interaction, thought as a heavier meson exchange occurring before and/or after the pion exchange. In fig. 2 the exchange of a pion between correlated nucleons is shown, the wiggly line denoting a $G$-matrix obtained only from the repulsive short range interaction. Remarkably such an effect could be well described by means of seemingly completely different models, like, e.g., exotic quark configurations; within the present scheme it is simpler to identify and understand the microscopical origin of the channel dependence of the effect we are looking for.

If $\mathcal{V}_{\pi}(r)$ denotes the one pion exchange potential in configuration space, the four diagrams of fig. 2 are well simulated by $g(r) \mathcal{V}_{\pi}(r), g(r)$ being the pair correlation function [more precisely we should evaluate the matrix element of $\mathcal{V}_{\pi}$ with correlated wave functions, i.e., solutions of the Bethe-Goldstone equation, instead making use of plane waves [44]: the previous parametrization corresponds to the static limit].

Within this scheme an overcounting of diagrams arises when two successive correlated pions are exchanged (as it happens in all but the first diagrams of fig. 11 when the first [diagrams d) and e)] or second [diagrams b) and c)] term only of the RPA series is considered). The diagrams presenting overcounting are displayed in fig. 3 .

In the static limit, however, two successive meson exchanges are $\propto g(r)^{2} \mathcal{V}_{\pi}^{2}(r)$, while dropping the double counting roughly corresponds to $\propto g(r)^{\frac{3}{2}} \mathcal{V}_{\pi}^{2}(r)$. Thus, due to the form of $g(r)$, the effect of the over counting is to amplify the suppression of the short range part of $V_{\pi}(r)$, i.e., in Fourier transforms, of its high momentum components. This outcome is unavoidable from a formal point of view: it originates from handling an effective interaction as a true potential.

Brown et al. suggested for $g(r)$ the very simple form

$$
g(r)=1-j_{0}\left(q_{c} r\right)
$$

(with $q_{c} \sim m_{\omega}$ ) which leads to the following expression of the correlated potential in Fourier transforms: 
$g(r) \mathcal{V}_{\pi}(r) \Longrightarrow \mathcal{V}_{\pi}(\mathbf{q})-\int \frac{d^{3} k}{(2 \pi)^{3}} \frac{2 \pi^{2}}{q_{c}^{2}} \delta\left(|\mathbf{k}|-q_{c}\right) \mathcal{V}_{\pi}(\mathbf{k}-\mathbf{q})$

widely employed in the literature 445] for two main reasons: it gives a good description of the elastic pionnucleus scattering [46,47] and it corresponds, in the Landau limit and accounting also for the correlated $\rho$ exchange, to a value for $g^{\prime} \sim 0.65$, consistent with many model-independent analysis. Furthermore, it cuts down the high momentum components of the correlated potential because of the cancellation between the two terms in (26) when $|\mathbf{q}| \gg q_{c}$.

We shall adopt in the following a different form - maintaining however the same philosophy - by writing the potential in the isovector spin-longitudinal channel as

$$
V_{\pi}(q)=\frac{f_{\pi N N}^{2}}{m_{\pi}^{2}}\left\{g_{L}^{\prime}(\mathbf{q})-\frac{\mathbf{q}^{2}}{m_{\pi}^{2}+\mathbf{q}^{2}}\right\} v_{\pi}^{2}\left(q^{2}\right) ;
$$

and in the isovector spin-transverse as

$$
V_{\rho}(q)=\frac{f_{\pi N N}^{2}}{m_{\pi}^{2}}\left\{g_{T}^{\prime}(\mathbf{q})-C_{\rho} \frac{\mathbf{q}^{2}}{m_{\rho}^{2}+\mathbf{q}^{2}}\right\} v_{\rho}^{2}\left(q^{2}\right) .
$$

A momentum dependence has been attributed to $g_{L}^{\prime}$ and $g_{T}^{\prime}$, such that

$$
g_{L}^{\prime}(\mathbf{q}) \stackrel{q \rightarrow \infty}{\longrightarrow} 1 \quad g_{T}^{\prime}(\mathbf{q}) \stackrel{q \rightarrow \infty}{\longrightarrow} C_{\rho} .
$$

Actually the functional forms we have chosen are

$$
\begin{aligned}
& g_{L}^{\prime}(q)=1+\left(g_{0}^{\prime}-1\right)\left[\frac{q_{c L}^{2}}{q_{c L}^{2}+q^{2}}\right]^{2} \\
& g_{T}^{\prime}(q)=C_{\rho}+\left(g_{0}^{\prime}-C_{\rho}\right)\left[\frac{q_{c T}^{2}}{q_{c T}^{2}+q^{2}}\right]^{2} .
\end{aligned}
$$

With these expressions we obtain the required high momenta cancellations and we force the same Landau limit $g_{0}^{\prime}$ in both channels, as it should be. Following [45 47] the value of $q_{c L}$ should be chosen around $m_{\omega}$, but, because of the previous considerations about the double counting problem we suggest as an acceptable compromise a slight increase of the value of $q_{c L}$ to $q_{c L} \sim 0.8 \div 0.9 \mathrm{GeV} / \mathrm{c}$ : in this way we effectively remove the over counting problem, without altering the low- $q$ behaviour of the effective interaction. We have no phenomenological indications about the precise value $q_{c T}$ should have: surely it should lie between $\sim 1 \mathrm{GeV} / \mathrm{c}$ and $\sim 2 \mathrm{GeV} / \mathrm{c}$. The lower limit comes when considering that obviously the effect of the SRC for the $\rho$-meson exchange should be sensible at shorter distances with respect to $1 / m_{\rho}$; the upper one is roughly determined by the inverse size of the $N N \rho$-vertex. Apart from these wide limits we assume $q_{c T}$ essentially as a free parameter.

Finally we come to the isoscalar spin-transverse channel. As we are going to discuss in the following, phenomenology does not impose severe constraints on the $\omega$-meson propagation in nuclear matter. Thus we simply assume

$$
V_{\omega}(q)=-\frac{f_{\pi N N}^{2}}{m_{\pi}^{2}} C_{\omega} \frac{\mathbf{q}^{2}}{m_{\rho}^{2}+\mathbf{q}^{2}} v_{\omega}^{2}(q) .
$$

The form factors in (27), (28) and (32) are in the usual dipole form

$$
v\left(q^{2}\right)=\frac{\Lambda_{m}^{2}-m_{m}^{2}}{\Lambda_{m}^{2}+\mathbf{q}^{2}-q_{0}^{2}}
$$

with cut-offs, respectively, chosen to be $\Lambda_{\pi}=1.3 \mathrm{GeV} / \mathrm{c}$ $\Lambda_{\rho}=2.5 \mathrm{GeV} / \mathrm{c}$ and $\Lambda_{\omega}=1.5 \mathrm{GeV} / \mathrm{c}$.

Finally let us discuss the coupling constants. We put as usual $f_{\pi N N}^{2} / 4 \pi=0.08$ and $f_{\pi N \Delta}^{2} / 4 \pi=0.32$; the value we use for $f_{\pi N \Delta}$ is commonly employed in the literature, somehow higher than the value obtained by the $N-N$ phase shift analysis carried out in constructing the Bonn potential, but lower than the results of a recent fit 48]. It is remarkable that the precise value of this parameter depends on the details of the underlying dynamical model: within the Bonn potential approach, in our opinion, is necessary to underestimate the $\pi N \Delta$ coupling, to effectively account for the $\Delta-\Delta$ repulsion inside a box diagram [49,50]. Concerning the best fit of Ref. [48], the higher value of the coupling constant was accompanied by a reduction of the cut-off so that the net effect is roughly the same.

Coming to vector mesons, we write the relativistic interaction lagrangian in the form

$$
g_{V} \bar{\psi} \gamma_{\mu} \psi \phi_{V}^{\mu}+\frac{f_{V}}{4 m} \bar{\psi} \sigma_{\mu \nu} \psi\left(\partial^{\mu} \phi_{V}^{\nu}-\partial^{\nu} \phi_{V}^{\mu}\right)
$$

which reduces, in the non relativistic limit, to

$$
i \frac{\left(f_{V}+g_{V}\right)^{2}}{4 m} \psi^{\dagger} \boldsymbol{\sigma} \times \mathbf{q} \psi \cdot \boldsymbol{\phi}_{v} .
$$

Let us finally define $C_{V}$ as

$$
\frac{\left(f_{V}+g_{V}\right)^{2}}{4 m}=C_{V} \frac{f_{\pi N N}^{2}}{m_{\pi}^{2}}
$$

so to give a meaning to eqs. (28) and (32).

Using the values given by Ref. [51] one gets $C_{\rho} \sim 2.3$. The coupling constant of the $\omega$ is not well known. Höler et al. [52] values correspond to $0.83<C_{\omega}<2.5$; Grein 53] and Grein and Kroll [54] provide respectively $C_{\omega}=$ 0.83 and 0.56 . Finally the $N-N$ phase shift analysis of Ref. [36] gives $C_{\omega}=1.5$, the value we have adopted in the present work. As it will become clear from our results the uncertainty over this parameter does not propagate too much on the final results, being the overall $\omega$-contribution small. Also the possible presence of a residual SRC interaction in this channel - that we do not include in the present calculation - should not influence the final results. 
Finally, the $\rho N \Delta$ coupling is also largely unknown. The analysis of Ref. [36] provides $C_{\rho}^{\Delta}=2.1$ but other values are also compatible. Here we have chosen $C_{\rho}=2.3$ both for $N$ and $\Delta$. The corresponding cut-offs are also chosen according to the Bonn potential parameters.

We remark that in the present approach the practically unknown $\Delta \Delta$-meson vertices are not required.

\section{EVALUATION OF THE DIAGRAMS}

The diagrams corresponding to the one loop corrections, described in Sect. II], are those of Fig. 11 (without $\Delta$ lines), or those with one $\Delta$ line in Fig. 4 and with two $\Delta$ lines in Fig. 5. Everywhere the wiggly line denotes a RPA-dressed meson $(\pi, \rho$ or $\omega)$ and a double solid line stands for a $\Delta$ propagator. Each term is a Feynman diagram, i.e., we could further reduce it in Goldstone diagrams by distinguishing all the possible time-orderings. Such a procedure entails an enormous increase in the number of terms required: for instance, diagrams a), b) and c) generate 24 Goldstone diagrams each, while 720 Goldstone diagrams come from d) and e) [remember that the internal mesonic lines are RPA dressed, so they are not forced to connect equal-times couples of points]. Since, furthermore, different particles may correspond to the internal mesonic lines, diagrams a), b) and c) must be evaluated for three different cases while d) and e) for nine: the explicit evaluation of all the separated Goldstone diagrams is a hopeless task.

We can, however, bypass this problem without loosing any information by means of the results of Refs. [33,34, where we caried out the analytic evaluation in momentum space of the functions $\Pi^{(3)}$ and $\Pi^{(4)}$ describing a fermionic loop with 3 or 4 external legs. We proved that only one $\theta$-function is truly necessary when dealing with the generic Feynman diagram $\Pi^{(n)}$ : the information carried by the remaining $\theta$ 's can be transferred to the analytical properties of the diagram in momentum space. Remarkably, this result can be proved by individually altering each Goldstone diagram, in such a way that their sum remains unaltered. Thus the information about the the single Goldstone diagram contributions are lost, but the overall Feynman diagram can be exactly evaluated. Furthermore it is possible to choose a particular energymomentum region where the analytical properties of the diagram are simply defined (this is typically a high energy region): there the loop integral can be explicitly carried out and then one is able to come back to the other kinematical regions through the study of the analytical properties of the whole diagram.

In momentum space we shall employ for the fermionic loops we are interested in the notation $\Pi^{(3)}(p, q)$ and $\Pi^{(4)}(p, q, k), p, q, k$ being the momenta entering the diagram, in clockwise order [the last momentum entering each diagram is obviously fixed by momentum conservation].
The function $\Pi^{(3)}\left(q_{1}, q_{2}\right)$, entering the diagrams d) and e), can be expressed as

$$
\begin{aligned}
\Pi^{(3)}\left(q_{1}, q_{2}\right)= & m^{2}\left[I^{3}\left(q_{1}, q_{2}\right)+I^{3}\left(-q_{1}, q_{2}-q_{1}\right)\right. \\
& \left.+I^{3}\left(-q_{2}, q_{1}-q_{2}\right)\right]
\end{aligned}
$$

where the function $I^{3}$ is in turn defined as

$$
\begin{aligned}
I^{3}\left(q_{1}, q_{2}\right) & =\frac{1}{(2 \pi)^{3} q_{1} q_{2} \sin ^{2} \chi}\left\{\left(y_{1} \cos \chi-y_{2}\right)\right. \\
& \cdot \log \frac{y_{1}-k_{F}}{y_{1}+k}+\left(y_{2} \cos \chi-y_{1}\right) \log \frac{y_{2}-k_{F}}{y_{2}+k} \\
& \left.-\sqrt{\Delta} \log \frac{y_{1} y_{2}-k_{F}^{2} \cos \chi+k_{F} \sqrt{\Delta}}{y_{1} y_{2}-k_{F}^{2} \cos \chi-k_{F} \sqrt{\Delta}}\right\}
\end{aligned}
$$

In the last equation $q_{1,2}$ denotes the norm of the 3 -vector $\mathbf{q}_{\mathbf{1}, \mathbf{2}}$ and $\chi$ is the angle between them. Furthermore

$$
y_{1,2}=\frac{m q_{1,2}^{0}}{q_{1,2}}-\frac{q_{1,2}}{2}
$$

and

$$
\begin{aligned}
G^{2} & =y_{1}^{2}-2 y_{1} y_{2} \cos \chi+y_{2}^{2} \\
\Delta & =G^{2}-k_{F}^{2} \sin ^{2} \chi .
\end{aligned}
$$

The diagrams a), b) and c) require two particular cases of $\Pi^{(4)}$, which can be expressed in terms of $\Pi^{(3)}$, namely

$$
\begin{aligned}
\Pi^{(4)}(k, q,-k)= & -\frac{m^{3}}{\mathbf{k} \cdot \mathbf{q}}\left\{\Pi^{(3)}(k, q)+\Pi^{(3)}(-k,-q)\right. \\
& \left.-\Pi^{(3)}(k,-q)-\Pi^{(3)}(-k, q)\right\} \\
\Pi^{(4)}(k, q,-q)= & \left(\frac{\partial}{\partial k^{0}}+\frac{\partial}{\partial q^{0}}\right) \Pi^{(3)}(k, q) .
\end{aligned}
$$

The reader is referred to [33,34] for further details on the analytical extension of the formulae.

The diagrams f) to $\mathrm{k}$ ) can be evaluated analytically too, if the approximation

$$
\frac{m_{\Delta} m}{m_{\Delta}-m} \gg \frac{k_{F}^{2}}{2 m}
$$

is assumed to hold: needless to say at the usual saturation density this is near to be exact. The corresponding formulae are not given here for obvious reasons of brevity. The reader is again referred to [34], where all the details are given: the analytical functions required in this case are as simple as the former ones.

The relevant point is that all the fermionic loop integrations have been now analytically performed, resulting in easily manageable functions. Thus the calculation of diagrams a) to $\mathrm{k}$ ) reduces to a 4-dimensional integral over a known analytical function (actually the integral is 3-dimensional because the integration over the azimuthal angle is trivial) and the sum of the many thousands of 
Goldstone diagrams required by our theoretical scheme is translated to the sum of 51 Feynman diagrams each one expressed as a 3-dimensional integral [the possible exchange of $\pi, \rho$ and $\omega$ being accounted for].

Explicitly diagram a) reads

$$
\Pi^{(a)}(k)=i \operatorname{Tr} \Gamma_{e m} \Gamma_{i} \Gamma_{e m} \Gamma_{i} \int \frac{d^{4} q}{(2 \pi)^{4}} \Pi^{(4)}(k, q,-k) V_{i}(q)
$$

with $\Gamma_{e m}=\left(1+\tau_{3}\right) / 2 ; \Gamma_{i}$ and $V_{i}$ are the vertex and the potential pertaining to the exchanged mesons; diagrams b) and c) read respectively

$$
\Pi^{(b)}(k)=i \operatorname{Tr} \Gamma_{e m} \Gamma_{i} \Gamma_{i} \Gamma_{e m} \int \frac{d^{4} q}{(2 \pi)^{4}} \Pi^{(4)}(k, q,-q) V_{i}(q)
$$

and

$$
\Pi^{(c)}(k)=\Pi^{(b)}(-k)
$$

Finally diagrams d) and e) are given by

$$
\begin{array}{r}
\Pi^{(d)}(k)=\int \frac{d^{4} q}{(2 \pi)^{4}} \operatorname{Tr} \Gamma_{i} \Gamma_{e m} \Gamma_{j} \Pi^{(3)}(q, k) V_{i}(q) \\
V_{i}(q+k) \operatorname{Tr} \Gamma_{j} \Gamma_{e m} \Gamma_{i} \Pi^{(3)}(-k,-q)
\end{array}
$$

and

$$
\begin{array}{r}
\Pi^{(e)}(k)=\int \frac{d^{4} q}{(2 \pi)^{4}} \operatorname{Tr} \Gamma_{i} \Gamma_{e m} \Gamma_{j} \Pi^{(3)}(q, k) V_{i}(q) \\
V_{i}(q+k) \operatorname{Tr} \Gamma_{i} \Gamma_{e m} \Gamma_{j} \Pi^{(3)}(-q,-k)
\end{array}
$$

where we explicitly indicated that two different particles ( $i$ and $j$ ) can be exchanged. Diagrams f) to $\mathrm{k}$ ) have the same structure of eqs. (48) and (49), but the proper expressions for the various $\Pi^{(3)}$ functions must be used [34, according to which line in the diagram pertains to a nucleon or to a $\Delta$.

The last step before the numerical evaluation of the integrals is the calculation of the spin-isospin traces. In the channels we are considering the various $\Gamma$ 's are respectively $\boldsymbol{\sigma} \cdot \mathbf{q} \tau_{\mu},(\boldsymbol{\sigma} \times \mathbf{q})_{i} \tau_{\mu}$ and $(\boldsymbol{\sigma} \times \mathbf{q})_{i}$ for $\pi, \rho$ and $\omega$-emission respectively. For the case of a $N \Delta$ transition vertex, the matrices $\boldsymbol{\sigma}$ and $\tau_{\mu}$ will be replaced by the proper spin-isospin transition ones, usually denoted by $\mathbf{S}$ and $T_{\mu}$.

The traces reduce to a coefficient in front of the diagram, including, if case, an angular dependence. Those pertaining the diagrams a), b) and c) are given in table I. where the contributions of the isoscalar and of the isovector part have been separated. Diagrams d) to $\mathrm{k}$ ) require the study of 72 different cases, because in each of them we must distinguish the particles exchanged inside (9 possibilities). We can simplify the situation observing that the diagrams can be characterized as follows:
1. the directions of the momentum flow in the two loops of diagrams d), f), h), j) are opposite (we shall call these "direct correlation diagrams"), while for e), g), i), k) case they are the same ("exchange correlation diagrams");

2. the diagrams are characterized by the exchanged particles, $\pi, \rho$ and $\omega$ : we must keep in mind their momentum space behaviour, i.e., pseudoscalar $(\mathrm{P})$ or vector $(\mathrm{V})$ character and the isospin $(0$ or 1$)$;

3 . we must also account for the number of $\Delta$ 's in the internal lines: $n_{\Delta}=0,1$ or 2 .

Then the traces can be factorized as

$$
\lambda T_{i j}^{I} S_{\mu \nu}
$$

where $S$ results from the spin part and reads

$$
\begin{gathered}
S_{P P}=4[\mathbf{q} \cdot(\mathbf{q}+\mathbf{k})]^{2} \\
S_{P V}=S_{V P}=S_{V V}=4\left[q^{2} k^{2}-(\mathbf{q} \cdot \mathbf{k})^{2}\right]
\end{gathered}
$$

$T$ comes from the isospin traces and holds

$$
\begin{aligned}
T_{00}^{T=0}=4 & T_{00}^{T=1}=0 \\
T_{01}^{T=0}=T_{10}^{T=0}=0 & T_{01}^{T=1}=T_{10}^{T=1}=4 \\
T_{11}^{T=0}=12 & T_{11}^{T=1}=\mp 8
\end{aligned}
$$

where the sign in $T_{11}^{T=1}$ is minus for the direct diagrams and plus for the exchange ones; finally $\lambda$ depends upon the number of $\Delta$ 's:

$$
\begin{aligned}
& \lambda^{T=0}=\left(\frac{4}{9}\right)^{n_{\Delta}} \\
& \lambda^{T=1}=\left(-\frac{2}{9}\right)^{n_{\Delta}} .
\end{aligned}
$$

Finally we note that in the diagrams f) to k) an $\omega$ cannot be exchanged: this reduces to 51 the number of diagrams effectively needed.

\section{RESULTS}

In this section we present the results of our calculation for the longitudinal response, which, in the infinite nuclear matter limit, is related to the polarization propagator

$$
\Pi^{\mu \nu}(x, y)=<\Psi_{0}\left|T\left\{j^{\mu}(x), j^{\nu}(y)\right\}\right| \Psi_{0}>
$$

by

$$
\begin{aligned}
R_{L}(k, \omega) & =-\frac{1}{\pi} \frac{Z}{\rho} G_{E p}(k) \Pi^{00}(k, \omega) \\
& =-\frac{1}{\pi} \frac{Z}{\rho} G_{E p}(k) \int d(x-y) e^{i k \cdot(x-y)} \Pi^{00}(x-y),
\end{aligned}
$$


$\rho$ being the nuclear density.

We have shown in the previous sections that, with the help of the analytical results from Refs. [33, 34, the computational problem reduces to the evaluation of one three-dimensional integral for each diagram; the numerical integration remains, however, highly delicate because the functions $\Pi^{(n)}$ exhibit a large number of singularities. Moreover, the large predicted cancellations between different terms require a great numerical precision.

The parameters used in the calculation are, unless otherwise specified, $g_{0}^{\prime}=0.5, q_{c L}=800 \mathrm{MeV} / \mathrm{c}, q_{c T}=1100$ $\mathrm{Mev} / \mathrm{c}$ and $k_{F}=1.36 \mathrm{fm}^{-1}$; we compare the results with the data of Meziani et al [7, 8] on $C a^{40}$ and $C^{12}$.

In fig. 6 we present the pion contribution to diagram a), singling out the first order term from the remaining part of the RPA series, to stress the role of RPA-dressing

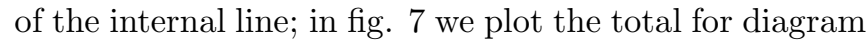
a), as well as the contributions from the different mesons. The relevance of the $\rho$-or, more correctly, of the effective interaction in the isovector spin-transverse channel - is evident: this feature persists in the other diagrams.

Next we come to the self-energy diagrams [b) and c)]. In fig. 8 we present the pion part, singling out first order and RPA corrections both for diagrams b) and c); we remark that a large cancellation occurs between them (they should cancel exactly in the case of a constant potential). In fig. 9 we display the total of b) and c), again singling out the terms from different mesons.

Concerning the self-energy diagrams a peculiar difficulty arises, namely the instability of the calculation at the edges of the response region; the reason is that these terms contain a self-energy insertion on the fermionic lines: but this is only the first term of a Dyson series. We know 'ab initio' that summing the whole series would correspond to a modification of the dispersion relation for the nucleon and, consequently, of the response region itself. It is not surprising then that the inclusion of the first term of the series only gives rise to wild oscillations: the convergence of the loop expansion is warranted by the smallness of the 1-loop contribution with respect to the mean field one: this is clearly not the case just around the edges of the response region. On the other hand the inclusion of the whole Dyson series for the nucleons is inconsistent with the loop expansion scheme and it should lead to the violation of sum rules and general theorems.

The sharpness of the Fermi surface is responsible for a large part of the problem: from the mathematical point of view, in fact, the diagram diverges when a pole in the denominator comes to the boundary of the integration region, and this happens just because the Fermi surface is sharp: this is not the case for a finite nucleus, thus the singularity could be removed by smearing somewhat the Fermi surface. We stressed before that such a procedure would require an enormous increase of the computational difficulties, so we attributed the nucleon a small finite width at momenta near the Fermi surface, to simulate the same effect. This correction is not included in figs. 8 and 9 but it will be accounted for in the final result.
Next we come to diagrams d) and e). Here $\pi, \rho$ and $\omega$ can contribute in all possible combinations. The single terms are shown in fig. 10, where we have plotted the sum of diagrams d) and e) separating the two pions, two $\rho$ 's, two $\omega$ 's and finally the mixed $(\pi \rho, \pi \omega$ and $\rho \omega)$ contributions. Fig. 11 displays instead separately diagrams d) and e). The effect of the $\Delta$ 's in the intermediate states is illustrated in fig. 12, where we reported the total contributions without, with one and two $\Delta$ 's. It is remarkable the strong cancellation occuring between the contributions from diagrams b) and c), which would strongly quench the response, and the ones from the other diagrams featuring the opposite. Finally we present separately the total from exchange, self-energy and correlation diagrams in fig. 13.

We evaluated all the results presented until now always using the set of parameters given at the beginning of this chapter, with the coupling constants and cutoffs of sect. [II]. Before presenting the comparison between our theoretical calculation and the experimental data we examine the sensitivity of the results to small changes of the parameters. Keeping the coupling constants and cutoffs as fixed, we display in fig. 14 the effect of variations of $g^{\prime}$, $k_{F}$ and of the many-boy cutoffs $q_{c L, T}$.

Finally we compare with the experimental data in figs. 15 and 16 for ${ }^{40} \mathrm{Ca}$ and ${ }^{12} \mathrm{C}$ respectively. In the second case we assume $k_{F}=1.2 \mathrm{fm}^{-1}$ to effectively take into account of the relevant smearing of the surface in such a light nucleus.

\section{DISCUSSIONS AND OUTLOOK}

In the previous section we presented the results of our calculation with a given choice of the model parameters. Now we need a critical analysis of these outcomes.

As a first point, let us come back to our theoretical approach to the many-body problem. The loop expansion automatically entails the fulfillment, order-by-order, of general theorems, sum-rules and so on. This because the loop expansion is a, if case asymptotic, well-behaves series of powers in a suitably chosen parameter, i.e., the coefficient multiplying the action, to be identified at the end of the calculation with $\hbar$. It is remarkable that, unlike the usual application of SPA, in our case the formal parameter of the expansion does not simply coincide with $\hbar$, because the Hubbard-Stratonovich transformation makes the effective bosonic action itself $\hbar$ dependent. This implies, in turn, that if the value of a given sum-rule does not depend on $\hbar$, then this sum-rule must be fulfilled at the mean field level: contributions coming from higher orders must separately vanish. This is the case, for instance, for the usual $f$ sum-rule, which reads:

$$
\int_{0}^{\infty} d \omega R_{L}^{T=0}(q, \omega)=\frac{q^{2}}{2 M}
$$

if in $R_{L}^{T=0}(q, \omega)$ the electromagnetic nucleonic form fac- 
tors are omitted. In fact the FFG longitudinal response fulfills eq. (60); the contributions from quantum fluctuations must then vanish, thus realizing explicitly the expected cancellations between Feynman diagrams at the same order. We verified the $f$ sum-rule in order to check the numerical accuracy and the inner consistency of our approach. The task is complicated because this sum-rule is energy weighted, i.e., contributions of the high-energy tale is emphasized. This requires, first of all, to extend the integration well beyond the QEP region.

We considered the two extreme cases $q=300$ and $410 \mathrm{MeV} / \mathrm{c}$ and then we extended the integration up to $\omega=400 \mathrm{MeV}$, cfr. Figs. 15-19, obtaining a saturation of the sum-rule of the order of $90 \%$ in both cases. To be more specific, at $q=300 \mathrm{MeV} / \mathrm{c}$, the exact value of the sum-rule is $q^{2} / 2 M=47.97 \mathrm{MeV}$ : with the integral extended up to $\omega=200 \mathrm{MeV}$ - containing anyway the whole region of the QEP - we obtain from l.h.s. of eq. (60) a value of $39.16 \mathrm{MeV}$, while extending the integral up to $\omega=400 \mathrm{MeV}$ we get a sum-rule value of $42.39 \mathrm{MeV}$. At $q=410 \mathrm{MeV} / \mathrm{c}$, extending the integral up to $\omega=400 \mathrm{MeV}$, we obtain a value of $80.47 \mathrm{MeV}$ to be compared with $q^{2} / 2 M=89.60 \mathrm{MeV}$. We consider this result quite satisfactory because it is clearly beliavable that the remaining contribution from the tale will saturate completely the $f$ sum-rule. We remark anyway that we estimate a numerical uncertainty of the calculation of the order of $2 \%$.

Coming to the detailed shape of the response, we observe that, a posteriori, the one-loop corrections to the mean field results are sizable, but still remarkably smaller than the mean field itself; this is not a trivial point, because the mesons included in the model strongly interact with the nuclear matter: then very large corrections, mainly for the case of the $\rho$-exchange, could well be expected - and this is the case indeed for some diagrams. But large cancellations among them are present and the final answers are reasonably small, indicating that the loop expansion works properly at this order. We expect, in fact, the loop expansion to be an asymptotic series and only an explicit evaluation to a given order can establish if it is still in the convergence region or not. Of course even an estimate of the two-loop corrections seems presently too hard to be performed.

The previous statement, i.e., that the approximation scheme works well at the one-loop order, requires however to be somehow weakened, because, as already remarked, we expect a failure of the first order calculations near the edges of the QEP: in fact our approach is not able to provide a shift of the whole peak, which is instead induced by the dressing of the nucleon in the medium, i.e. by rewriting the nucleon propagator as

$$
\frac{1}{p_{0}-\frac{p^{2}}{2 m} \pm i \eta} \rightarrow \frac{1}{p_{0}-\frac{p^{2}}{2 m}-\Sigma\left(p, p_{0}\right)}
$$

Our scheme contains, in fact, diagrams with one selfenergy insertion [diagrams b) and c)], but the whole
Dyson's series should be summed up to shift the position of QEP. Thus we expect to account for the strength of the peak but

1. the position of the peak should remain around the free one;

2. at the border of the QEP almost all the response is generated by the infinite series of self-energy insertions summed up through the Dyson's equations. Since only the first term of this series is present, at the edges of the FFG response region the loop expansion necessarily fails.

Just to give the reader a visual feeling of how a simple shift could affect the response, we have reported in fig. 17 one of the results of fig. 16, but shifting the curve of $30 \mathrm{MeV}$ upward. With this (formally unjustified) trick the agreement with the data becomes impressive, opening an interesting question: clearly the corrections stemming from the loop expansion resonably describe, in a channel dependent way, the photoexcitation mechanism. This was our main purpose, but on the other hand this approximation fails to describe the mechanism leading to the nuclear binding, as it is clearly shown in fig. 17. This should be expected, but leaves the question: "Could someone put all the things together?"

What discussed above concerned the theoretical framework. Now we come to the dynamical model employed in the present work. This topic is not so well grounded on formal properties of field theory, but of course is much more strictly linked with the physical domain: the model, as it comes out clearly from our discussion, is not parameter-free. Many parameters are not experimentally well determined. Our choice was to keep the coupling constants and the cut-off as near as possible to commonly accepted values, using the Bonn potential as guideline for those quantities for which we have no sufficiently clear experimental evidence. In this way the number of parameters is drastically reduced. Even $g^{\prime}$ can vary only between narrow limits (i.e. $g^{\prime}=0.5 \div 0.6$ in non static conditions 41]) and for $k_{F}$ we made the choice $k_{F}=1.36 \mathrm{fm}^{-1}$ for ${ }^{40} \mathrm{Ca}$ and $k_{F}=1.2 \mathrm{fm}^{-1}$ for ${ }^{12} C$, which seems quite natural.

The many-body cut-off $q_{c L}$ can also vary, reasonably, between 800 and $900 \mathrm{MeV} / \mathrm{c}$, while, finally, the cut-off on the spin-transverse channel has been treated as an approximately free parameter. Between these really narrow constraints we have chosen the most favourable situation to reproduce the experimental data of [8].

We observe that the choice of $k_{F}$ and $g^{\prime}$ seems to be quite reasonable, while, according to the discussion of sect. III a rather higher value for $q_{c L}$ (like for instance $q_{c L}=900 \mathrm{MeV} / \mathrm{c}$ ) should be preferable. The net effect should be a small reduction of the quenching of the quasi elastic peak.

Very recently a new analysis of the Rosenbluth separation [13], based on experimental data taken at the Bates 
Laboratory, seemed to indicate a reduction of the longitudinal response quite lower than the one previously believed. The discrepancies between the two results indicate that more careful experimental analyses are required. It is of course outside our scope to discuss the validity of the experimental data: we only present here a comparison of the two sets of results, together with our theoretical outcomes obtained with two different sets of parameters, namely $g^{\prime}=0.5, q_{c L}=800 \mathrm{MeV} / \mathrm{c}$, $q_{c T}=1100 \mathrm{MeV} / \mathrm{c}$ and $g^{\prime}=0.6, q_{c L}=900 \mathrm{MeV} / \mathrm{c}$, $q_{c T}=1200 \mathrm{MeV} / \mathrm{c}$ (see fig. 18). The first set better approaches the results of Saclay, while the second is more suitable to reproduce the Bates data, at least in the left side of the peak.

To summarize we believe to have shown that, within a mesonic theory, a 1-loop calculation provides a channel dependent photo-excitation mechanism able to significantly quench the longitudinal response. The size of this quenching is, however, not completely determined, both theoretically and experimentally. In the present model the major uncertainty comes from the effective interaction in the $\rho$-channel: combined changes in $g^{\prime}, q_{c T}$ and $\Lambda_{\rho}$ can significantly affect the response - without modifying, however the trend illustrated so far. Exclusive $\left(\mathrm{ee}^{\prime}, 2 \mathrm{~N}\right)$ experiments are required to improve our understanding of the p-h effective interaction 42 and to better fix the parameters of the model. Coming to the perspectives opened by the present approach, the next step will be, obviously, to extend the calculation to the transverse (magnetic) response making use of the same theoretical scheme: the number of diagrams to be evaluated increases rapidly, because both the MEC and the direct photo-excitation of the $\Delta$ are present. In particular, the possible electromagnetic excitation of $\Delta$-h pairs can be viewed as a channel dependent vertex renormalization in the medium - thus coming back to the idea of M. Ericson and M. Rosa-Clot 24] - and could be well responsible for the different behaviour of the longitudinal and transverse channel. Remarkably this feature emerges at the 1-loop level. Unfortunately, some new parameters - the meson$\Delta \Delta$ couplings - will enter the game. This calculation is presently in progress.

More response functions can be evaluated along these lines, like, e.g., the isoscalar-spin longitudinal and transverse ones [56]. Here an interesting opportunity is offered, because direct $\Delta$ excitation is forbidden in both channels. Thus, if our guess about the relevance of the $\Delta$ excitation in simultaneously explaining the electromagnetic responses is correct, the separation of the spin logitudinal and transverse responses in the $S=1, T=0$ channel should give rise to less pronounced differences.

Furthermore, the same model can be applied to the parity-violating responses [57, where a good confidence in the microscopical model is needed to establish the feasibility of experiments able to detect the $\gamma-Z_{0}$ interference in the electron scattering.

Moreover, as far as higher momentum data will become available, other issues will have increasing relevance: first of all the study of relativistic effects, at least those coming the relativistic kinematics of the nucleons.

[1] J.L. Friar and M. Rosen, Ann. Phys. 87:289, 1974.

[2] G. Cò and J. Heisenberg, Phys. Lett. 197B:489, 1987.

[3] M. Traini, Phys. Lett. 213B:1, 1988.

[4] M. Traini, S. Turck-Chièze and A. Zghiche, Phys. Rev. 34C:2799, 1988.

[5] R. Altemus et al., Phys. Rev. Lett., 44:965, 1980.

[6] P. Barreau et al. Nucl. Phys., A402:515, 1983.

[7] Z. E. Meziani et al. Phys. Rev. Lett., 52:2180, 1984.

[8] Z. E. Meziani et al. Phys. Rev. Lett., 54:1233, 1985.

[9] C. Marchand et al. Phys. Lett., 153B:29, 1985.

[10] M. Deady et al. Phys. Rev., C33:1897, 1986.

[11] G. C. Blatchley et al. Phys. Rev., C34:1243, 1986.

[12] P. Quinn et al. Phys. Rev., C37:1609, 1988.

[13] T.C. Yates et. al., Phys. Lett. 312B:382, 1993.

[14] W. M. Alberico, M. Ericson and A. Molinari. Ann. Phys., 154:356, 1984.

[15] S. Boffi, C. Giusti and F. D. Pacati. Phys. Rep., 226C:1, 1993.

[16] A. Dellafiore, F. Lenz and F. A. Brieva. Phys. Rev., C31:1088, 1985.

[17] F. A. Brieva and A. Dellafiore. Phys. Rev., C36:899, 1987.

[18] M. Cavinato et al. Nucl. Phys., A423:376, 1984.

[19] S. Drodz, G. P. Cò, J. Wambach and J. Speth. Phys. Lett., B185:287, 1987.

[20] G. P. Cò, K. F. Quader, R. D. Smith and J. Wambach. Nucl. Phys., A485:61, 1988.

[21] J. V. Noble. Phys. Rev. Lett., 46:412, 1981.

[22] L. S. Celenza, A. Rosenthal and C. M. Shakin. Phys. Rev. Lett., 53:891, 1984.

[23] L. S. Celenza, A. Rosenthal and C. M. Shakin. Phys. Rev., C33:1012, 1986.

[24] M. Ericson and M. Rosa-Clot. Zeit. Phys., A324:373, 1986.

[25] W. M. Alberico, R. Cenni, A. Molinari and P. Saracco. Ann. of Phys., 174:131, 1987.

[26] W. M. Alberico, R. Cenni, A. Molinari and P. Saracco. Phys. Rev. Lett., 65:1845, 1990.

[27] W. M. Alberico, R. Cenni, and A. Molinari. Prog. in Part. and Nucl. Phys., 23:171, 1989.

[28] R. Cenni, E. Galleani, F. Napoli, P. Saracco and M. Sassetti. Feynman integrals in theoretical, nuclear and statistical mechanics. Bibliopolis, Naples, 1989.

[29] R. Cenni and P. Saracco. Nuovo Cimento, D11:303, 1989.

[30] G. Morandi, E. Galleani d'Agliano, F. Napoli and C. Ratto. Adv. Phys., 23:867, 1974.

[31] H. Keiter. Phys. Rev., 2B:3777, 1970.

[32] H. Kleinert. Fort. Phys., 26:565, 1978.

[33] R. Cenni and P. Saracco. Nucl. Phys., A487:279, 1988.

[34] R. Cenni, F. Conte, A. Cornacchia and P. Saracco. La Rivista del Nuovo Cimento, vol. 15 n. 12, 1992.

[35] K. Holinde. Phys. Rep., C68:121, 1981.

[36] R. Machleidt, K. Holinde and Ch. Elster. Phys. Rep., 
C149:1, 1987.

[37] A. B. Migdal. Ž. Ėksp. Teor. Fiz., 61:2210, 1971.

[38] A. B. Migdal. Rev. Mod. Phys., 50:107, 1978.

[39] W.H. Dickoff, A. Faessler, J. Meyer-ter-Vehn and H. Müther, Phys. Rev. 23C:1154, 1981.

[40] W.H. Dickoff, A. Faessler, J. Meyer-ter-Vehn and H. Müther, Nucl. Phys. 368A:445, 1981.

[41] R. Cenni and P. Saracco, Phys. Lett. 246B:315, 1990.

[42] R. Cenni. To be published on the Proceedings of the Mainz Workshop on the European Electron Facility, Mainz October 7 - 9, 1992.

[43] G. E. Brown, S. O. Bäckman, E. Oset and W. Weise. Nucl. Phys., A286:191, 1977.

[44] R. Cenni and G. Dillon. Nuovo Cimento Lett., 30:138, 1981.

[45] E. Oset and L. L. Salcedo. Nucl. Phys., A468:631, 1987.

[46] E. Oset and W. Weise. Nucl. Phys., A319:365, 1979.

[47] E. Oset and W. Weise. Nucl. Phys., A329:47, 1979.

[48] R. Cenni, G. Dillon and P. Christillin. Nuovo Cimento, A97:9, 1987.

[49] R. Cenni, F. Conte and U. Lorenzini. Phys. Rev., C39:1588, 1989.

[50] R. Cenni, Md. A. Matin and P. Saracco. Nuovo Cimento, A104:1753, 1991

[51] G. Höler and E. Pietarinen. Nucl. Phys., B95:210, 1975.

[52] G. Höler et al. Nucl. Phys., B114:505, 1976.

[53] W. Grein. Nucl. Phys., B131:255, 1977.

[54] W. Grein and P. Kroll. Nucl. Phys., A338:332, 1980.

[55] W. M. Alberico, A. De Pace, A. Drago and A. Molinari. La Rivista del Nuovo Cimento, 14-n.5:, 1991.

[56] R.J. Peterson, private communications.

[57] M.J. Musolf, T.W. Donnelly, J. Dubach, S.J. Pollock, S. Kowalski and E.J. Beise, CEBAF Preprint \#TH-93-11, June 1993.

\section{FIGURE CAPTION}

FIG. 1. One loop diagrams. (a) exchange diagram, (b) and (c): self-energy diagrams, (d) and (e): correlation diagrams.

FIG. 2. Diagrams inducing SRC on the pion exchange.

FIG. 3. Diagrams with overcounting in two successive correlated pion exchanges.

FIG. 4. Diagrams (f), (g), (h) and (i) [see text] with one $\Delta$ line.

FIG. 5. Diagrams (j) and (k) [see text] with two $\Delta$ lines.
FIG. 6. Contribution to $R_{L}$ of the $\pi$-exchange, diag. a), for ${ }^{40} \mathrm{Ca}$ at $q=300 \mathrm{MeV} / \mathrm{c}$. Dotted line: first order, dashed lines: remaning part of RPA, solid line: total.

FIG. 7. Contribution to $R_{L}$ of the $\pi, \rho$ and $\omega$-exchanges, diag. a), for ${ }^{40} \mathrm{Ca}$ at $q=300 \mathrm{MeV} / \mathrm{c}$. Dotted line: $\pi$, dashed line: $\rho$, solid line: $\omega$.

FIG. 8. Contribution to $R_{L}$ of the $\pi$-exchange, diag. b) and c), for ${ }^{40} \mathrm{Ca}$ at $q=300 \mathrm{MeV} / \mathrm{c}$. Dotted line: first order diag. b), thick dots: first order diag. c); dashed line: RPA corrections diag. b), dash-dotted line: RPA corrections diag.c); solid line: total.

FIG. 9. Contribution to $R_{L}$ of the $\pi, \rho$ and $\omega$-exchanges, diag. b) and c), for ${ }^{40} \mathrm{Ca}$ at $q=300 \mathrm{MeV} / \mathrm{c}$. Pion: dotted line; $\rho$ : dashed line, $\omega$ : dash-dotted line; total: solid line.

FIG. 10. Contribution to $R_{L}$ from diags. d) and e) for ${ }^{40} \mathrm{Ca}$ at $q=300 \mathrm{MeV} / \mathrm{c}$. Dots: $\pi \pi$, dashes: $\rho \rho$, dash-dots: $\omega \omega$, dots with stars: $\pi \rho$, dashes with stars: $\pi \omega$, dash-dots with stars: $\rho \omega$; solid: total.

FIG. 11. Contribution to $R_{L}$ from diags. d) and e) for ${ }^{40} \mathrm{Ca}$ at $q=300 \mathrm{MeV} / \mathrm{c}$. diagrams d): dashed line; diagrams e): dash-dotted line; total: solid line.

FIG. 12. Contribution to $R_{L}$ from diags. d) to k) for ${ }^{40} \mathrm{Ca}$ at $q=300 \mathrm{MeV} / \mathrm{c}$. no $\Delta$ 's, diags. d) and e): dashed line; one intermediate $\Delta$, diagrams f), g), h) and i): dash-dotted line; two intermediate $\Delta$ 's, diagrams j) and k): dotted line; total: solid line.

FIG. 13. Contribution to $R_{L}$ from diags. a) to $\mathrm{k}$ ) for ${ }^{40} \mathrm{Ca}$ at $q=300 \mathrm{MeV} / \mathrm{c}$. Contribution of exchange diagram a): dashed line; self-energy diagrams b and c): dash-dotted line; correlation diagrams d) to $\mathrm{k}$ ): dotted line; total: solid line.

FIG. 14. Sensitivity of the one-loop contribution to $R_{L}$ to the many-body parameters. All parameters but the ones explicitly indicated are those previously stated. Solid line: standard parameters; dashed line: $g^{\prime}=0.6$, dotted line: $k_{F}=1.2 \mathrm{fm}^{-1}$, dash-dotted line: $q_{L}=900 \mathrm{MeV} / \mathrm{c}, q_{T}=1200$ $\mathrm{MeV} / \mathrm{c} . R_{L}$ for ${ }^{40} \mathrm{Ca}$ is displayed in $\mathrm{MeV}^{-1} \times 1000$ versus the transferred energy (in $\mathrm{MeV}$ ). 
FIG. 15. Comparison of $R_{L}$ with experimental data [taken from ref. [8] for ${ }^{40} \mathrm{Ca}$ as a function of the energy (in $\mathrm{MeV}$ ). The dashed line refers to the Free fermi gas contibution, the solid line represents the total results, while in the dash-dotted line the contribution of the diags. without internal $\Delta$ lines [diags. f) to k)].

FIG. 16. As fig. 15 but data are taken from ${ }^{12} C$ and here $k_{F}=1.2 \mathrm{fm}^{-1}$.

FIG. 17. As fig. 15, but the whole curve has been shifted [see text for explanation]. Only the case $q=300 \mathrm{MeV} / \mathrm{c}$ is plotted.

FIG. 18. Response functions compared with the data of ref. 8] and 13. Solid line: results with $q_{c L}=800 \mathrm{MeV} / \mathrm{c}$ and $q_{c T}=1100 \mathrm{MeV} / \mathrm{c}$; dashed line: results with $q_{c L}=900$ $\mathrm{MeV} / \mathrm{c}$ and $q_{c T}=1200 \mathrm{MeV} / \mathrm{c}$.

\section{TABLES}

\begin{tabular}{c|c|c|c|}
\hline \hline Particle & Channel & Diag. a) & Diag. b) \\
$\pi$ & $T=0$ & $12 q^{2}$ & $12 q^{2}$ \\
\cline { 2 - 4 } & $\mathrm{T}=1$ & $-4 q^{2}$ & $12 q^{2}$ \\
\cline { 2 - 4 } & e.m. & $2 q^{2}$ & $6 q^{2}$ \\
& $T=0$ & $24 q^{2}$ & $24 q^{2}$ \\
\hline & $T=1$ & $-8 q^{2}$ & $24 q^{2}$ \\
\hline & e.m. & $4 q^{2}$ & $12 q^{2}$ \\
& $T=0$ & $4 q^{2}$ & $8 q^{2}$ \\
\hline \hline
\end{tabular}

TABLE I. Table of the spin-isospin traces for the diagrams a) and b) (diagram c) coincides with b)) 